\title{
A New Type of Streaming in Pulse Tubes
}

\author{
W. Liang and A.T.A.M. de Waele \\ Department of Applied Physics, Eindhoven University of Technology \\ Eindhoven, The Netherlands
}

\begin{abstract}
By numerical simulation a new type of streaming in pulse tubes has been found. The geometry in the simulation consists of the pulse tube with its flow straighteners. The straighteners are described as a porous medium. Viscous effects are taken into account, but heat transfer with the wall is not considered. The gas flow in the pulse tube is periodic. Positive and negative boundary conditions are imposed which result in flow fields with opposing directions. The net flow field is obtained by the superposition of these two flow fields. It turns out that two loops are formed in the tube. This paper analyzes this phenomenon and explains how the flow resistance of the straightener and the viscous effect of the tube wall result in this type of streaming.
\end{abstract}

\section{INTRODUCTION}

In this paper we determine the three-dimensional phenomena in pulse tubes such as mass streaming and turbulence by numerical simulation using the CFD software CFX-5.7. In the geometry studied narrow tubes are connected to the in- and outlet of the pulse tube. This can lead to jets that are reduced by flow straighteners made of a stack of fine screens placed at both ends of the pulse tube. The stack of screens forms a porous medium. The flow in porous media is calculated by a generalization of the Navier-Stokes equations and of Darcy's law. After the straightener the velocity in the radial direction is reduced and the velocity in z-direction becomes practically homogenous along the cross-section. The number of the screens that is required to erase the jet effectively can be calculated by Eq. (1) ${ }^{1}$

with

$$
N \geq \frac{\Delta p_{B}}{\Delta p_{A}}
$$

$$
\Delta p_{B}=\frac{1}{2} \rho \dot{V}^{2}\left[\left(\frac{1}{A_{1}}\right)^{2}-\left(\frac{1}{A_{2}}\right)^{2}\right] .
$$

Here $\Delta p_{A}$ is the pressure drop when gas flows through a single screen, $\dot{V}$ is the volume flow, $A_{1}$ is the cross-sectional area of the connection tube, and $A_{2}$ is the cross-sectional area of the pulse tube.

The flow in the pulse tube is periodic in time. The frequency is determined by a rotary valve (in the case of GM-type PTR's) or by the compressor (for Stirling-type PTR's). In the first part of 


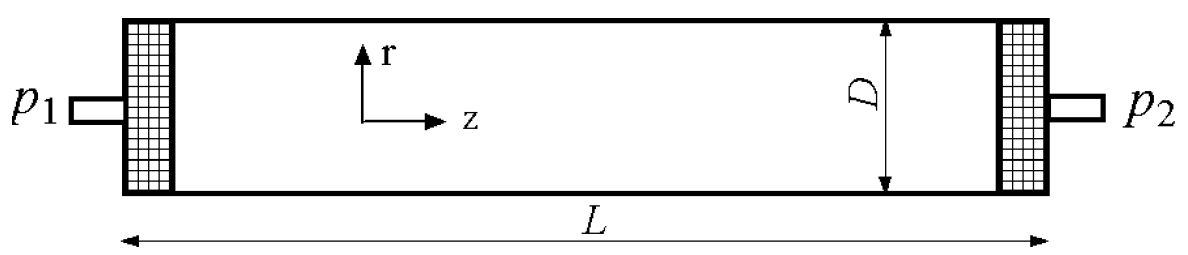

Figure 1. Geometry of the system under consideration.

this paper quasi-DC flow is used to study the flow in the pulse tube. In this model the geometry and boundary conditions are symmetrical and the combined effect of the flow straightener and tube wall is considered by numerical simulation. We report a type of streaming in the pulse tube which leads to a thermal short-circuit inside the tube. The basic reason for the effect is that the gas, leaving the flow straightener, flows differently from the gas entering the flow-straightener. The numerical results presented in this paper show this phenomenon. We also describe the situation of laminar flow with approximate analytical relations. Finally we give the results in the case of a harmonic pressure oscillation.

\section{NUMERICAL PROCEDURE}

In order to study the effect of the tube wall, the so-called BSL Reynolds model is chosen. BSL is a Reynolds Stress model based on the $\omega$-equation. ${ }^{2,3}$ The advantage of the $\omega$-equation is that it allows for a more accurate near-wall treatment with an automatic switch from a wall function to a low-Reynolds number formulation based on the grid spacing. The flow through the straightener is also calculated numerically. For this paper it is sufficient that the flow of the gas leaving a flow straightener is practically homogeneous. For the simulation we imposed the following conditions:

- The relative error of the velocities is less than $5 \times 10^{-5}$.

- The domain imbalance, defined as $\varepsilon=\left(S_{\text {in }}-S_{\text {out }}\right) / S_{\text {in }}\left(S_{\text {in }}\right.$ is the mass, momentum, or energy flowing into the domain and $S_{\text {out }}$ the corresponding quantity flowing out) should be less than $1 \%$. The domain imbalance is used to check the convergence of the solution. Small values of the domain imbalance indicate that conservation has been maintained.

- The number of grids in the boundary layer must be 10 or more. For low-Reynolds flow 15 or more grids are required.

- The variable $\mathrm{Y}_{+}=\Delta n \sqrt{\tau_{\mathrm{w}} / \rho} / v$ (where $\Delta n$ is the distance between the first and second grid point off the wall, $\tau_{w}$ is the wall shear stress, and $v$ is kinematic viscosity) is a dimensionless distance from the wall. It is a very important parameter to check whether the mesh is fine enough to solve the boundary layer and the possibility that the simulation can converge properly. In our case, $\mathrm{Y}_{+}$(for low-Reynolds flow) should be less than two. ${ }^{2}$

All results shown in this paper satisfy these conditions. The error in the velocity is smaller than $5 \times 10^{-5}$, the domain imbalance is less that $3 \mathrm{ppm}, 16$ grids are in the boundary layer, and $\mathrm{Y}_{+}<1.8$.

In the simulation the working fluid is real helium at $273 \mathrm{~K}$ and $10 \mathrm{bar}$, and the surface of the tube wall is smooth. The gas flow leaving the flow straightener is practically homogeneous. After the gas enters the pulse tube the viscous effect of the tube wall will influence the flow field.

As shown in Fig. 1 the model pulse-tube has two straighteners, one at each end. We consider mainly a tube with a diameter $D$ of $4 \mathrm{~mm}$ and a length $L$ of $40 \mathrm{~mm}$. The thickness of the straighteners is $2 \mathrm{~mm}$. It should be mentioned that $\Delta p=p_{2}-p_{1}$ is the pressure difference over the entire system including the flow straighteners. Most of the pressure drop is over the flow straighteners. The pressure variations inside the tube are many order of magnitude smaller that this value.

At first the high pressure is at the left and low pressure at the right $\left(p_{1}=p_{\mathrm{H}}\right.$ and $\left.p_{2}=p_{\mathrm{L}}\right)$. The resulting velocity field is calculated. Then we reverse the high and low pressure $\left(p_{1}=p_{\mathrm{L}}\right.$ and $\left.p_{2}=p_{\mathrm{H}}\right)$ and get the opposite velocity field. Next we add the two velocity fields and get the net velocity field. In the two situations the pressure in the tube is practically the same, so the fluid density in the tube is the same in both cases. By adding the velocity fields we also get the mass flux distribution. 


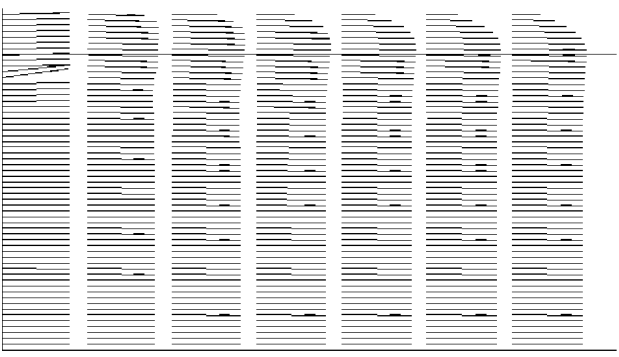

(a)

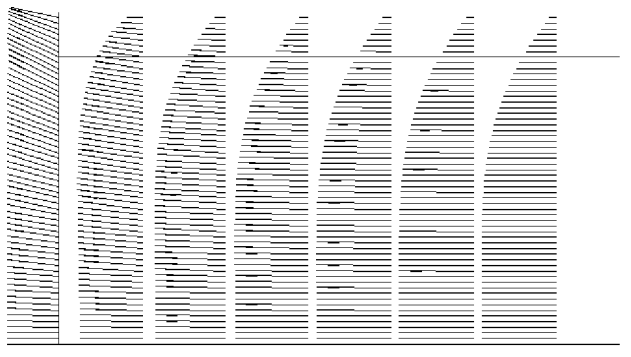

(b)

Figure 2. Velocity fields close to the flow straightener. a) flow to the right. The velocity right at the exit of the flow straightener is $3.89 \mathrm{~m} / \mathrm{s}$; b) flow to the left.

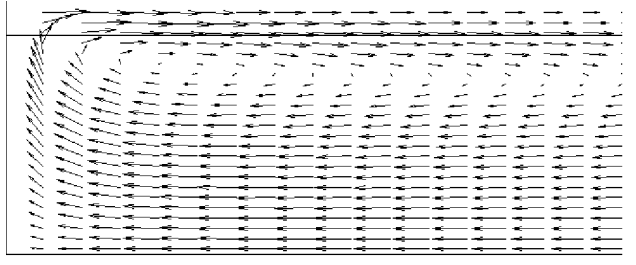

(a)

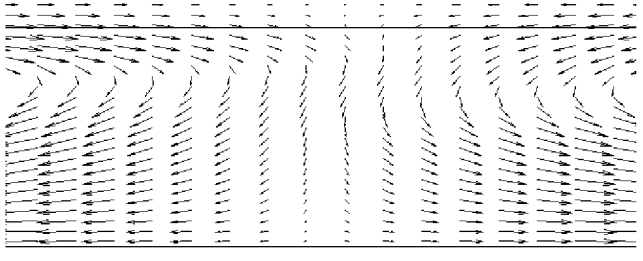

(b)

Figure 3. Net velocity fields. a) close to the flow straightener; b) in the centre of the tube.

\section{RESULTS}

Figure 2 gives results for $\Delta p=0.1$ bar, $D=4 \mathrm{~mm}$, and $L=40 \mathrm{~mm}$. In Fig. 2 a the gas flows to the right. After the straightener the flow is homogenous and the boundary layer near the wall is very thin. Downstream, the boundary layer becomes thicker. In Fig. $2 \mathrm{~b}$ the gas flows to the left. In this case the boundary layer, which is large close to the straightener, is damaged when gas is about to enter the straightener.

In the case of Fig. 2 the maximum pressure variation inside the tube is $19 \mathrm{~Pa}-$ thus, about three orders of magnitude less that the overall pressure difference.

We add the positive and the negative flow fields to obtain the net velocity field, which is the streaming effect under consideration. Fig. 3a shows the streaming near the straightener. The plug flow in one direction and the boundary-layer flow in the opposite direction lead to a flow distribution with a net non-zero flow close to the wall and opposing flow in the centre of the tube. The direction of the net velocity near the tube wall is from the straightener to the middle of the tube, while the direction of the net velocity in the center of the tube is from the middle to the straightener. Due to the symmetry of the system the streaming in the middle of the pulse tube must be zero. Fig. $3 \mathrm{~b}$ shows the streaming near the middle of the tube. Two closed circuits of gas flow are formed in each half of tube (see Fig.4). For larger diameters the intensity becomes smaller due to the reduced influence of the wall.

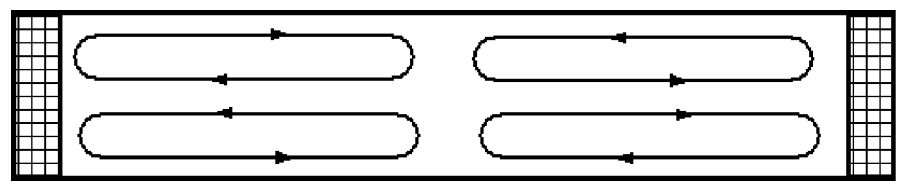

Figure 4. Schematic drawing of the net circulation in the tube. Two vortex rings are formed inside the tube. 


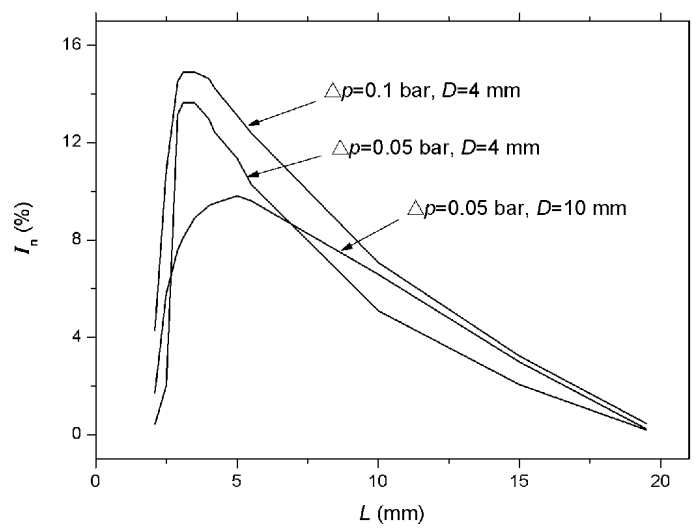

Figure 5. Profiles of the relative net mass flow in the tube. The corresponding pressure drops and the tube diameters are indicated with the curves.

In order to get a measure of the magnitude of the effect we introduce the relative net mass flow defined as

with

$$
I_{\mathrm{n}}=\frac{v_{\mathrm{n}}}{v_{0}}
$$

and

$$
v_{\mathrm{n}}=\int \mathrm{d} A\left(v_{+}+v_{-}\right) / 2
$$

$$
v_{0}=\int \mathrm{d} A v_{+},
$$

where $v_{\mathrm{n}}$ is the net velocity and $v_{0}$ the average velocity. Fig. 5 shows $I_{\mathrm{n}}$ in three different situations. In all cases there is a maximum value of $I_{\mathrm{n}}$ along the pulse tube. Normally it is located at the transition distance. The curves with the same diameter, but different $\Delta p$ are almost the same. If the tube diameter is changed from $4 \mathrm{~mm}$ to $10 \mathrm{~mm}$, the intensity is weaker because the gross mass flow is larger in this case. The distribution of the net mass flow related to the ratio of the diameter and length of the tube will be investigated in the future.

\section{LAMINAR-FLOW APPROXIMATION}

For laminar flow the velocity in the $z$-direction is, in good approximation, given by the relation $^{4}$

$$
v_{\mathrm{z}}(r, z)=v_{0}(z)\left[1-\exp \left(1-0.332 \xi-0.075 \xi^{2}\right)\right]
$$

with

$$
\xi^{2}=(R-r)^{2} \rho v_{0} /(\eta z)
$$

with $R$ the tube radius, $r$ the radius, $\rho$ the fluid density, $v_{0}(z)=v_{\mathrm{z}}(0, z)$ the velocity in the center of the tube, and $\eta$ the fluid viscosity. At the exit of the flow straightener the velocity $v_{00}=v_{z}(0,0)$ is given by

$$
\dot{\mathrm{m}}=\pi R^{2} \rho v_{00} \text {. }
$$

Furthermore

$$
\dot{\mathrm{m}}=\rho \int_{0}^{R} 2 \pi r v_{\mathrm{z}} \mathrm{d} r
$$

so

$$
v_{0}(z) \int_{0}^{R} 2 \pi r v_{\mathrm{z}} \mathrm{d} r=R^{2} v_{00} \text {. }
$$



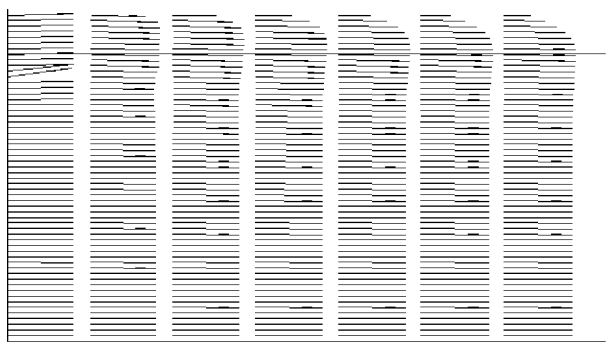

(a)

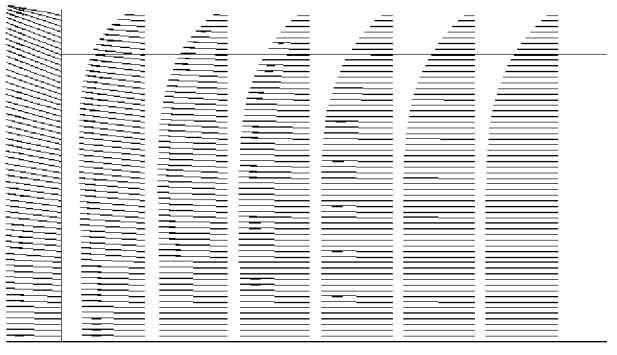

(b)

Figure 6. Case of harmonic pressure oscillations. The figure shows the velocity fields close to the flow straightener: a) flow to the right; b) flow to the left.

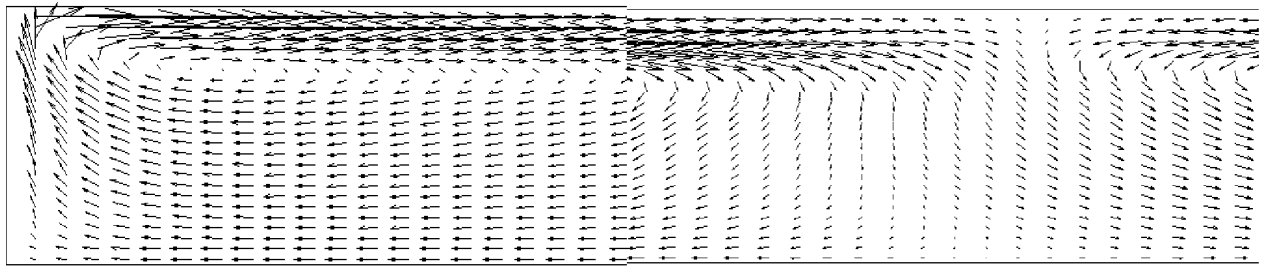

(a)

(b)

Figure 7. Net velocity fields: a) close to the flow straightener; b) close to the middle of the tube.

This relation implies that the velocity in the center is enhanced due to the reduced velocity near the wall. The net flow, obtained by adding the velocity fields in the positive and the negative directions, shows the structure as shown in Fig. 3.

\section{HARMONIC PRESSURE OSCILLATIONS}

In the previous part of this paper we considered steady flows in the two directions. However, in a real PTR we have oscillating flow and the situation is never steady. In principle this can lead to very complicated situations where the boundary layer has no time to develop. Therefore we also considered the case of a harmonic pressure oscillation. We applied a boundary condition according to $p_{1}=p_{0}+p_{\mathrm{a}} \cos \omega t$ and $p_{2}=p_{0}-p_{\mathrm{a}} \cos \omega t$. Figure 6 gives the flow fields and Fig. 7 the net result. As can be seen, the main features are the same as in Figs. 2 and 3.

\section{DISCUSSION}

The unsymmetrical flow is caused by the combined effect of the straightener and the viscous drag of the tube wall. It forms two closed circuits for the mass flow in the pulse tube. The circulation can reduce the performance of the pulse tube refrigerator because it mixes the gases with different temperatures. The fact that two cells are developed which basically have no interaction, makes the estimation of the overall effect on the performance of the system interesting but complicated.

In our analysis heat transfer between the gas and the tube wall was not considered. Furthermore the working fluid in this analysis is real helium at $273 \mathrm{~K}$. The streaming will be weaker at lower temperatures because the viscosity of the helium is smaller. The phenomenon will be investigated in more detail in the future.

\section{ACKNOWLEDGEMENTS}

This work is supported by STW, the Dutch Technology Foundation. 


\section{REFERENCE}

1. I. Tanaeva, Low-temperature cryocooling, thesis, Department of Applied Physics, Eindhoven University of Technology (2005), p.40.

2. Turbulence and Wall Function Theory, Handbook of CFX 5.7.1, pp. 68-69.

3. Turbulence and Near-Wall Modeling, Handbook of CFX 5.7.1, p. 104.

4. L.D. Landau and E.M. Lifshitz, Fluid Mechanics, $2^{\text {nd }}$ edition, Pergamon Press (1987), p.161. 